\title{
Diagnostic Differences in Expert Second-Opinion Consultation Cases at a Tertiary Sarcoma Center
}

\author{
Asha Rupani, ${ }^{1}$ Magnus Hallin, ${ }^{1}$ Robin L. Jones, ${ }^{1,2}$ Cyril Fisher, ${ }^{2,3}$ and Khin Thway $\mathbb{D}^{1,2}$ \\ ${ }^{1}$ Sarcoma Unit, Royal Marsden Hospital, London SW3 6JJ, UK \\ ${ }^{2}$ The Institute of Cancer Research, London SW3 6JB, UK \\ ${ }^{3}$ Department of Musculoskeletal Pathology, Royal Orthopaedic Hospital NHS Foundation Trust, \\ Robert Aitken Institute for Clinical Research, University of Birmingham, Birmingham B15 2TT, UK \\ Correspondence should be addressed to Khin Thway; khin_thway@yahoo.com
}

Received 7 April 2020; Revised 11 August 2020; Accepted 16 September 2020; Published 29 September 2020

Academic Editor: Cornelis Verhoef

Copyright (c) 2020 Asha Rupani et al. This is an open access article distributed under the Creative Commons Attribution License, which permits unrestricted use, distribution, and reproduction in any medium, provided the original work is properly cited.

\begin{abstract}
Soft tissue tumors are diagnostically challenging, and it is recommended that these are reported or reviewed by specialist soft tissue pathologists. We present our experience with second-opinion (consultation) cases in a specialist tertiary sarcoma center. The aim of this study was to determine areas of diagnostic difficulty in soft tissue pathology. We assessed 581 second-opinion cases which were reviewed by two experienced pathologists in a period of one year. There was $62 \%$ concordance between the original and the second-opinion diagnosis, with diagnostic discrepancy in 38\%. The largest group of soft tissue neoplasms received for second opinion was fibroblastic/myofibroblastic tumors, and most major diagnostic problems were encountered in adipocytic and socalled "fibrohistiocytic" tumors. Major diagnostic errors impacting management were found in 148 cases (25\%). Morphologic assessment of tumors, judicious use of molecular techniques, newer immunostains and their interpretation, along with importance of knowledge of rarer entities were found to be most useful in avoiding errors.
\end{abstract}

\section{Introduction}

Soft tissue tumors are diagnostically challenging due to their rarity, swiftly-evolving histopathologic and molecular diagnostic classification, overlapping morphology and immunophenotype with many other neoplasms, and dependence on ancillary immunohistochemical and molecular investigations for many diagnoses. The UK National Institute for Health and Care Excellence (NICE) Guidelines indicate that all soft tissue tumors should be either reported or reviewed by soft tissue pathologists who regularly report them, participate in the UK National Soft Tissue Pathology External Quality Assurance scheme, and are core members of a Sarcoma Multidisciplinary Team [1]. The incidence of malignant soft tissue tumors is around 25-50 per million in the general population $[2,3]$. The Sarcoma Unit at the Royal Marsden Hospital, a tertiary, comprehensive cancer treatment center receives a total of approximately 3500 cases of soft tissue neoplasms every year $[4,5]$, comprising biopsies or resections performed at the center, referral material, and second-opinion/consultation cases. Referral cases are usually previously reported cases from other hospitals, sent when the patient is referred to a soft tissue specialist center for further management or clinical opinion. Second-opinion cases are distinct from referrals as they are usually sought directly by general pathologists for rarer and diagnostically challenging cases. Our center has previously audited diagnostic discrepancy rates and causes for referred cases of soft tissue neoplasms $[4,5]$, but there are few studies assessing diagnostic discrepancies for second-opinion cases in soft tissue pathology [6-9]. This is important, particularly now in an era where there is widespread routine use of ancillary molecular diagnostic modalities and immunohistochemical assays that can serve as correlates of molecular genetic alterations [10-12]. In this study, we assessed the areas of diagnostic discrepancy in the reporting of soft tissue neoplasms referred for expert opinion, to assess specific discrepancy patterns between general and specialist soft tissue pathologists. 


\section{Materials and Methods}

A retrospective audit was performed for second-opinion cases received in the Sarcoma Unit of the Royal Marsden Hospital. Cases were retrieved from the pathology secondopinion database. The cases were received as paraffin blocks, slides, or a combination of both, depending on the local policies of the referring institution, with a covering letter from the referring pathologist and usually the original report. The second opinion was given in the form of a letter addressed to the referring pathologist. Details from each case were obtained from the hospital electronic patient record, or from the hospital pathology document retrieval system to which completed documents are scanned. Original reports or descriptions from the referring pathologists were compared with the second-opinion diagnosis for differences in diagnosis, and, where comparable, other parameters such as grading and use of additional ancillary techniques were recorded. In cases where differential diagnoses were offered by the referring pathologist, the most favoured diagnosis was used for the purpose of analysis. Grading was assigned according to the system by the French Federation of Cancer Centers Sarcoma Group (FNCLCC) $[13,14]$.

The cases were categorized according to differentiation, and behavior was categorized as malignant, intermediate, or benign based on the World Health Organization classification (2013) of tumors of soft tissue and bone [3]. Minor discrepancies can be those of diagnosis, classification, or grading, where the discrepancy was not thought to provoke significant management change, whereas major discrepancies are defined as those that could instigate significant change in clinical management, resulting in under- or overtreatment. Major discrepancies can be divided into six categories: (1) malignant $\longrightarrow$ malignant (resulting in significant management change), (2) malignant $\longrightarrow$ benign, (3) benign $\longrightarrow$ malignant, (4) mesenchymal $\longrightarrow$ nonmesenchymal, (5) other (e.g., benign $\longrightarrow$ benign, but resulting in significant management change), and (6) major grading discrepancies, comprising tumors in which there was any interchange of grade between grades 2-3 and grade 1 (as this could lead to change in management) $[4,5]$. The groups in this study were divided into (1) malignant to malignant, (2) malignant to benign, (3) malignant to intermediate, (4) benign to benign, (5) benign to malignant, (6) benign to intermediate, (7) intermediate to intermediate, (8) intermediate to benign, and (9) intermediate to malignant. The reasons for discrepancy were analyzed, and challenging groups of cases are discussed along with some of the diagnostic pitfalls. The results were also compared to the two previous audits from this center addressing referral cases $[4,5]$.

\section{Results}

A total of 581 cases were received for second opinion in the one-year (2012) period assessed. 360 cases (62\%) showed no change in diagnosis and 221 cases (38\%) showed a difference between the original diagnosis and the second-opinion diagnosis (summarized in Table 1). Tumors with fibroblastic/myofibroblastic differentiation comprised the largest group of neoplasms received for second opinion (99 cases, 17\%). The diagnosis was concordant in 65 cases, with a difference in opinion in 34 cases (34\%) (Table 2). Adipocytic tumors comprised the next largest group of soft tissue neoplasms received for second opinion and showed significant discordance between the original and second opinion diagnoses. There were 74 cases, of which 32 (43\%) showed diagnostic discrepancy (Table 3). Smooth muscle tumors represented the third largest group with 35 cases, of which discrepancy was seen in $10(28 \%)$ (Table 4). The next group was of vascular tumors with 32 cases, of which discrepancy was noted in $10(31 \%)$ (Table 5). Nerve sheath tumors also comprised 32 cases, with discrepancy in 11 (34\%) (Table 6). The next group was of skeletal muscle tumors with 14 cases, with discrepancy in two (10\%). A case of benign fibrous histiocytoma was diagnosed as spindle cell rhabdomyosarcoma on morphological appearances. The second case with differential diagnosis of solitary fibrous tumor (SFT) and synovial sarcoma was diagnosed as spindle cell rhabdomyosarcoma on morphology and negative TLE1 immunohistochemistry. The group of socalled "fibrohistiocytic" tumors included 25 cases, of which the diagnosis was discrepant in 16 (64\%) (Table 7). In one case with uncertain diagnosis, a second opinion diagnosis of fibrohistiocytic neoplasm of uncertain malignant potential was offered.

Analysis was also performed between the broad categories of malignant, intermediate, and benign soft tissue neoplasms, as change in classification of behavior can have impact on patient management (as summarized in Table 8). 285 cases (49\%) were received for second opinion from academic/university teaching hospitals, while 242 (42\%) were from community (district general) hospitals, 38 from private clinics, and 16 from overseas university/teaching hospitals. The percentage of concordance of diagnosis based on the type of referring institute is shown in Table 9.

\section{Discussion}

Soft tissue neoplasms represent a heterogeneous group of tumors with a wide range of clinical behaviors. Prognostication and appropriate treatment, including with targeted therapies, is dependent on accurate diagnosis and behavioral stratification. Because of the increasing subspecialization of surgical pathologists, there is a tendency for less frequent exposure of general pathologists to soft tissue neoplasms. As sarcomas comprise only $1 \%$ of all adult cancers, even experienced general pathologists see insufficient numbers to gain familiarity or expertise. The error rate in histopathological diagnosis of soft tissue sarcomas in the recent literature is between $14 \%$ and $47 \%$, which reiterates the importance of obtaining further opinion from a specialist soft tissue pathologist for these neoplasms [6-9].

Arbiser et al. reviewed 500 cases of soft tissue lesions referred for second opinion. There was diagnostic agreement in $68 \%$, with major discrepancy in $25 \%$ and minor 
TABLE 1: Summary of second-opinion cases received according to assigned referral and second-opinion lineage/differentiation.

\begin{tabular}{|c|c|c|c|c|}
\hline Tumor categories with second-opinion diagnoses & Total no. of cases & $\begin{array}{l}\text { No discrepancy in } \\
\text { opinion }\end{array}$ & $\begin{array}{l}\text { Discrepancy in } \\
\text { opinion }\end{array}$ & $\%$ with discrepancy \\
\hline \multicolumn{5}{|l|}{ Specific lineage assignable } \\
\hline Fibroblastic/myofibroblastic & 99 & 65 & 34 & $34 \%$ \\
\hline Adipocytic & 74 & 42 & 32 & $43 \%$ \\
\hline Smooth muscle & 35 & 25 & 10 & $28 \%$ \\
\hline Vascular & 32 & 22 & 10 & $31 \%$ \\
\hline Nerve sheath & 32 & 21 & 11 & $34 \%$ \\
\hline Skeletal muscle & 14 & 12 & 2 & $14 \%$ \\
\hline So-called "fibrohistiocytic" & 25 & 9 & 16 & $64 \%$ \\
\hline Pericytic & 3 & 1 & 2 & $67 \%$ \\
\hline \multicolumn{5}{|l|}{ Lesions of uncertain differentiation } \\
\hline Synovial sarcoma & 9 & 6 & 3 & $33 \%$ \\
\hline Atypical fibroxanthoma & 7 & 5 & 2 & $28 \%$ \\
\hline Pleomorphic hyalinizing angiectatic tumor & 2 & 2 & 0 & - \\
\hline Myoepithelial tumors & 9 & 6 & 3 & $33 \%$ \\
\hline Ossifying fibromyxoid tumor & 4 & 3 & 1 & $25 \%$ \\
\hline Angiomatoid fibrous histiocytoma & 1 & 0 & 1 & $100 \%$ \\
\hline Intramuscular myxoma & 1 & 0 & 1 & $100 \%$ \\
\hline Acral fibromyxoma & 1 & 0 & 1 & $100 \%$ \\
\hline Epithelioid sarcoma & 3 & 1 & 2 & $67 \%$ \\
\hline Undifferentiated pleomorphic sarcoma & 31 & 16 & 15 & $48 \%$ \\
\hline $\begin{array}{l}\text { Spindle cell sarcoma (including postradiation } \\
\text { sarcoma) }\end{array}$ & 27 & 13 & 14 & $52 \%$ \\
\hline Undifferentiated round cell tumor & 9 & 5 & 4 & $44 \%$ \\
\hline Undifferentiated malignant neoplasm & 22 & 16 & 6 & $27 \%$ \\
\hline Bone and cartilage tumors/lesions & 7 & 4 & 3 & $43 \%$ \\
\hline \multicolumn{5}{|l|}{ Others } \\
\hline Follicular dendritic sarcoma & 2 & 2 & 0 & - \\
\hline Phyllodes tumor & 3 & 3 & 0 & - \\
\hline Gastrointestinal stromal tumor & 9 & 5 & 4 & $44 \%$ \\
\hline Specialised genital stromal tumors & 12 & 4 & 8 & $66 \%$ \\
\hline Olfactory neuroblastoma & 2 & 2 & 0 & - \\
\hline Ewing sarcoma & 3 & 3 & 0 & - \\
\hline Melanoma, including clear cell sarcoma & 20 & 11 & 9 & $45 \%$ \\
\hline PEComa, including lymphangioleiomyomatosis & 3 & 3 & 0 & - \\
\hline Meningioma & 1 & 0 & 1 & $100 \%$ \\
\hline Mesothelioma & 14 & 7 & 7 & $50 \%$ \\
\hline Carcinoma & 33 & 18 & 15 & $45 \%$ \\
\hline Germ cell/sex-cord stromal tumors & 8 & 6 & 2 & $25 \%$ \\
\hline Miscellaneous benign lesions & $24=134$ & $22=86$ & $2=48$ & $8 \%$ \\
\hline Total cases & 581 & 360 & 221 & $38 \%$ \\
\hline
\end{tabular}

discrepancy in $7 \%$, and total diagnostic discrepancy of $32 \%$ [6]. Their distribution of discrepant cases was of benign mesenchymal lesions diagnosed as sarcoma in $45 \%$, sarcoma as benign tumors in $23 \%$, nonmesenchymal lesions diagnosed as sarcoma in $20 \%$, and major grading discrepancies in $12 \%$. In our study, we found a discrepancy rate of $38 \%$ (Table 1). The first group included cases of spindle cell lipoma and fat necrosis diagnosed as liposarcoma, fasciitis diagnosed as sarcoma, and cellular fibrohistiocytic tumors and tenosynovial giant cell tumor diagnosed as sarcoma. The second group comprised sarcoma (not otherwise specified) (NOS) and leiomyosarcoma misdiagnosed as benign lesions. Nonmesenchymal lesions such as melanoma, carcinoma, and lymphoma were mistaken for sarcoma in the third group, particularly desmoplastic neurotropic melanoma misdiagnosed as malignant peripheral nerve sheath tumor (MPNST). In their experience as well as ours, with the exception of nonmesenchymal lesions, the diagnosis for all major discrepant cases could be made on the basis of the hematoxylin and eosin- (H\&E-) stained slides, and diagnostic error was due to the lack of familiarity of nonspecialist surgical pathologists with rare and unusual neoplasms [6]. In their study, $80.4 \%$ cases were from community hospitals, 9.6\% from academic medical centers and 5.6\% from international hospitals, $4.2 \%$ from commercial laboratories, and less than $1 \%$ from government institutions.

Lehnhardt et al., in their review of 603 patients with soft tissue neoplasms received for second opinion over seven years, analyzed the types of lesions presenting challenges, discrepancies in grading, and impact of specimen type and the referring institute on the accuracy of the original diagnosis [7]. Their most frequent second-opinion diagnosis was liposarcoma, malignant fibrous histiocytoma (MFH)/ sarcoma (NOS), synovial sarcoma, and leiomyosarcoma. 
TABLE 2: Fibroblastic/myofibroblastic tumors with discrepancy in opinion.

\begin{tabular}{|c|c|c|}
\hline Original diagnosis & Second-opinion diagnosis & Comments \\
\hline \multicolumn{3}{|l|}{ Malignant to malignant, 2 cases } \\
\hline Pleomorphic sarcoma & Myxoinflammatory fibroblastic sarcoma & \\
\hline Low-grade spindle cell neoplasm & $\begin{array}{l}\text { Low-grade fibromyxoid sarcoma/sclerosing } \\
\text { epithelioid fibrosarcoma }\end{array}$ & $\begin{array}{l}\text { MUC4 negative but FUS-CREB gene fusion } \\
\text { present }\end{array}$ \\
\hline \multicolumn{3}{|l|}{ Malignant to Intermediate, 6 cases } \\
\hline Low-grade fibromyxoid sarcoma & Fibromatosis & MUC4 negative and $\beta$-catenin positive \\
\hline Fibrosarcoma & Fibromatosis & MUC4 negative and $\beta$-catenin positive \\
\hline Gastrointestinal stromal tumor & Fibromatosis & $\begin{array}{l}\text { KIT and PDGFRA mutational analysis } \\
\text { negative and } \beta \text {-catenin positive }\end{array}$ \\
\hline Synovial sarcoma & Inflammatory myofibroblastic tumor & ALK gene rearrangements absent \\
\hline Sarcoma & Myxoinflammatory fibroblastic sarcoma & \\
\hline $\begin{array}{l}\text { High-grade sarcoma, possibly } \\
\text { gastrointestinal stromal tumor }\end{array}$ & Solitary fibrous tumor & $\begin{array}{l}\text { KIT and PDGFRA mutational analysis } \\
\text { negative and focal TLE1 positive }\end{array}$ \\
\hline \multicolumn{3}{|l|}{ Malignant to benign, 2 cases } \\
\hline Low-grade spindle cell tumor & Nodular fasciitis & \\
\hline Leiomyosarcoma & Mammary-type myofibroblastoma & \\
\hline \multicolumn{3}{|l|}{ Benign to benign, 13 cases } \\
\hline Fibrous hamartoma of infancy & Nodular fasciitis & \\
\hline Spindle cell tumor & $\begin{array}{c}\text { Fibroblastic tumor without malignant } \\
\text { features }\end{array}$ & ETV6 negative, $\mathrm{t}(8 ; 15)$ present \\
\hline Genital stromal fibroepithelial polyp & Superficial myofibroblastoma & \\
\hline Benign fibrous histiocytoma & Superficial myofibroblastoma & \\
\hline $\begin{array}{l}\text { Inflammatory myofibroblastic tumor-type } \\
\text { lesion }\end{array}$ & $\begin{array}{l}\text { Fibroblastic proliferation of unknown } \\
\text { significance }\end{array}$ & ALK and MUC4 immunostains negative \\
\hline Lipoma & Intravascular fasciitis & CDK4 immunostain negative \\
\hline Angiomyxoma & Fibroma & \\
\hline Benign lesion & Myofibroblastoma & \\
\hline Specialised genital stromal tumor & Myofibroblastoma & \\
\hline Neurothekeoma & Fibromyxoma & MUC4 negative \\
\hline Benign lesion & Pleomorphic fibroma & \\
\hline Nodular fasciitis & Myofibroma & \\
\hline Benign lesion & Fibroma & \\
\hline
\end{tabular}

Benign to malignant, 1 case

Proliferative myositis Possibility of low-grade fibromyxoid sarcoma

Benign to intermediate, 2 cases

Angiofibroma

Solitary fibrous tumor

Benign spindle cell lesion

Intermediate to intermediate, 1 case

Spindle cell neoplasm of uncertain malignant potential

Solitary fibrous tumor

Fibromatosis

Intermediate to benign, 5 cases

Fibromatosis

Palmar fibromatosis

Atypical nodular spindle cell lesion

Fibrous pseudotumor/solitary fibrous tumor

Fibromatosis

Intermediate to malignant, 2 cases

Solitary fibrous tumor

Dermatofibrosarcoma protuberans/ solitary fibrous tumor

Low-grade fibromyxoid sarcoma

Myxofibrosarcoma
Scar tissue

Ossifying fasciitis

Nodular fasciitis

Fibroma of testis

Proliferative myositis
MUCA negative and $\beta$ -

KIT and PDGFRA mutational analysis negative and $\beta$-catenin positive

ALK gene rearrangements absent

T and PDGFRA mutational analysis negative and focal TLE1 positive
They reported an overall discrepancy rate of $38.3 \%$. They had the lowest agreement in cases of leiomyosarcoma at $25.8 \%$ and MPNST at $21.6 \%$, which was not shared in this study (Table 1 ). In their study, in $1.4 \%$ of cases, the diagnosis was changed from malignant to benign and in $7.6 \%$ from benign to malignant. They reported concordant primary diagnosis in $28.3 \%$ for pathologists in private clinics, $29.6 \%$ for hospital pathologists, $36.8 \%$ for university hospital pathologists, and $70.5 \%$ for their own department [7]. In this current study, the concordance rate was $60 \%$ for cases from academic/university teaching hospitals and $60 \%$ from private clinics, compared with $54 \%$ for district general hospitals. 
TABLE 3: Adipocytic tumors with discrepancy in opinion.

\begin{tabular}{|c|c|c|}
\hline Original diagnosis & Second-opinion diagnosis & Comments \\
\hline \multicolumn{3}{|l|}{ Malignant to malignant, 12 cases } \\
\hline $\begin{array}{l}\text { Malignant peripheral nerve sheath } \\
\text { tumor }\end{array}$ & Dedifferentiated liposarcoma & MDM2 amplified \\
\hline Atypical neoplasm & Well-differentiated liposarcoma & MDM2 amplified \\
\hline Myxoid sarcoma & Dedifferentiated liposarcoma & MDM2 failed \\
\hline Pleomorphic leiomyosarcoma & Dedifferentiated liposarcoma & MDM2 amplified \\
\hline Leiomyosarcoma & Dedifferentiated liposarcoma & MDM2 amplified \\
\hline Sarcomatoid renal cell carcinoma & Dedifferentiated liposarcoma & \\
\hline Pleomorphic sarcoma & Dedifferentiated liposarcoma & \\
\hline Sarcoma & $\begin{array}{l}\text { Dedifferentiated liposarcoma with } \\
\text { rhabdomyosarcomatous areas }\end{array}$ & MDM2 amplified \\
\hline Leiomyosarcoma & Dedifferentiated liposarcoma & \multirow{2}{*}{$\begin{array}{c}\text { MDM2 amplified } \\
\text { MDM2 amplified, ALK negative, CDK4 focal, } \\
\text { INI1 + }\end{array}$} \\
\hline Undifferentiated sarcoma & Dedifferentiated liposarcoma & \\
\hline Low-grade sarcoma & Dedifferentiated liposarcoma & \multirow{3}{*}{$\begin{array}{l}\text { MDM2 not amplified in all cases, but CDK4 } \\
\text { positive in } 1 \text { case }\end{array}$} \\
\hline Undifferentiated sarcoma & Well-differentiated liposarcoma & \\
\hline \multicolumn{3}{|l|}{ Malignant to benign, 7 cases } \\
\hline 7 cases of liposarcoma & Lipoma with or without fat necrosis & \\
\hline \multicolumn{3}{|l|}{ Benign to benign, 5 cases } \\
\hline Spindle cell lipoma & Fibrolipoma & \multirow[t]{5}{*}{ MDM2 not amplified } \\
\hline Hamartoma & Fibrolipoma & \\
\hline Spindle cell lipoma & Lipoblastoma & \\
\hline Fibrous hamartoma of infancy & Lipoblastomatosis & \\
\hline Myxoma & Spindle cell lipoma & \\
\hline \multicolumn{3}{|l|}{ Benign to malignant, 5 cases } \\
\hline 4 lipomas, 1 stromal lesion & Well-differentiated liposarcoma & MDM2 amplified in all cases \\
\hline \multicolumn{3}{|l|}{ Intermediate to malignant, 2 cases } \\
\hline $\begin{array}{l}\text { Inflammatory myofibroblastic } \\
\text { tumor }\end{array}$ & Dedifferentiated liposarcoma & MDM2 amplified \\
\hline Solitary fibrous tumor & Dedifferentiated liposarcoma & $\begin{array}{l}\text { MDM2 amplified, CDK4 positive, MUC4, } \\
\text { DOG1, ALK negative }\end{array}$ \\
\hline Nondiagnostic material, 1 case & Dedifferentiated liposarcoma & MDM2 amplified \\
\hline
\end{tabular}

TABle 4: Smooth muscle tumors with discrepancy in opinion.

\begin{tabular}{lc}
\hline Original diagnosis & Second-opinion diagnosis \\
\hline Malignant to malignant, 5 cases & \\
Epithelioid rhabdomyosarcoma & Epithelioid leiomyosarcoma \\
Malignant spindle cell tumor & Cutaneous leiomyosarcoma \\
Myxofibrosarcoma & Leiomyosarcoma \\
Gastrointestinal stromal tumor & Leiomyosarcoma \\
Angiosarcoma & Leiomyosarcoma \\
\hline Benign to malignant, 1 case & \\
Cellular leiomyoma & Low-grade leiomyosarcoma \\
\hline Intermediate to malignant, 4 cases & \\
Smooth muscle tumor of uncertain malignant potential & Low-grade leiomyosarcoma \\
Atypical leiomyoma & Low-grade leiomyosarcoma \\
Smooth muscle tumor of uncertain malignant potential & Leiomyosarcoma, grade 2 \\
Smooth muscle tumor of uncertain malignant potential & Leiomyosarcoma \\
\hline
\end{tabular}

This could be explained from the current practices in the UK, where more complicated cases are usually managed in larger referral centers, which are also university teaching hospitals, and many of these have a pathologist with special interest in soft tissue pathology. This is usually not the case with the district general hospitals, which could explain the lower concordance rate.
Al-Ibraheemi and Folpe more recently analyzed secondopinion discrepancy rates for pediatric bone and soft tissue neoplasms. There was agreement in $71 \%$ of cases, while $21 \%$ had minor and $8 \%$ had major discrepancies [8]. Their study did not include the cases of undifferentiated neoplasms reported in this audit, reflecting higher incidences of such tumors in the adult population (Table 1). 
TABle 5: Vascular tumors with discrepancy in opinion.

\begin{tabular}{lc}
\hline Original diagnosis & Second-opinion diagnosis \\
\hline Malignant to malignant, 3 cases & Epithelioid angiosarcoma \\
$\begin{array}{l}\text { Poorly differentiated carcinoma } \\
\text { Poorly differentiated malignant neoplasm }\end{array}$ & $\begin{array}{c}\text { Epithelioid angiosarcoma } \\
\text { Angiosarcoma }\end{array}$ \\
Gastrointestinal stromal tumor & \\
\hline Malignant to benign, 1 case & Hemorrhage with hemangioma \\
Low-grade vascular lesion & Epithelioid hemangioendothelioma \\
\hline Malignant to intermediate, 1 case & Hemangioma \\
Metastasis of adenocarcinoma & Hemangioma \\
\hline Benign to benign, 2 cases & Low-grade angiosarcoma \\
Myofibroma & \\
Angiolipomatous tumor & Hemangioma \\
Benign to malignant, 1 case & Vpithelioid hemangioma \\
Intermediate to benign, 2 cases & Vascular proliferation and sclerosis \\
\hline Kaposiform hemangioendothelioma & \\
Atypical vascularised tumor of unknown malignant potential &
\end{tabular}

TABLE 6: Nerve sheath tumors with discrepancy in opinion.

\begin{tabular}{lc}
\hline Original diagnosis & Second-opinion diagnosis \\
\hline Malignant to malignant, 1 case & Malignant peripheral nerve sheath tumor \\
\hline Melanoma or less likely cellular schwannoma & Schwannoma \\
\hline Malignant to benign, 3 cases & Schwannoma/neurofibroma \\
\hline Low-grade sarcoma & Neurofibroma \\
\hline Low-grade spindle cell neoplasm & \\
\hline Spindle cell liposarcoma & Plexiform cellular schwannoma \\
\hline Benign to benign, 1 case & Myxoid atypical neurofibroma \\
\hline Neural tumor & Atypical granular cell tumor \\
\hline Benign to intermediate, 2 cases & Malignant schwannoma \\
\hline Spindle cell lesion & Low-grade MPNST \\
\hline Granular cell tumor & Malignant granular cell tumor \\
\hline Benign to malignant, 3 cases & Low-grade MPNST
\end{tabular}

MPNST: malignant peripheral nerve sheath tumor.

In a more recent study from France, Perrier et al. reported a discrepancy rate of $14 \%$, with higher probability of discordance for a final diagnosis of desmoid tumors in comparison to liposarcomas [9]. They performed a cost analysis and found that centralized histologic reviews are likely to provide cost savings compared to the cost of additional treatment in case of a wrong diagnosis. With the discordance rate of $38 \%$ in this study and major diagnostic errors in 148 cases, a wrong diagnosis means unnecessary treatment to the patient with additional financial burdens on the National Health Service.

There were insufficient cases in this study to analyze grading differences, particularly because the focus of a second-opinion consultation was for diagnosis rather than for reporting prognostic factors such as grading. Grading was not applicable in 465 cases (80\%) as they were benign or intermediate soft tissue neoplasms, nonmesenchymal tumors, and undifferentiated malignant neoplasms or showed discrepancy in diagnosis between the original diagnosis and the expert second opinion, making grading inappropriate. Although grading discrepancy assessment would have been relevant in 116 cases (20\%) of malignant soft tissue tumors, many sarcomas types are associated with aggressive behavior, such that grading was not mentioned in the original or second-opinion report. Grading was given (in the original or second opinion report) in 26 cases (5\%). Of these, the grading matched only in nine cases and was not mentioned in the original report in 13 or in the second-opinion report in four.

This study included 20 melanocytic lesions, 14 mesotheliomas, and 33 carcinomas amongst other nonmesenchymal lesions in the second-opinion diagnosis 
TABLE 7: Fibrohistiocytic tumors with discrepancy in opinion.

\begin{tabular}{lc}
\hline Original diagnosis & Second-opinion diagnosis \\
\hline Malignant to benign, 3 cases & \\
\hline Malignant spindle cell tumor & Giant cell tumor of soft parts \\
\hline Leiomyosarcoma & Fibrous histiocytoma \\
\hline Vascular lesion with malignant features & Epithelioid fibrous histiocytoma \\
\hline Benign to benign, 8 cases & Pigmented villonodular synovitis \\
\hline Clear cell soft tissue neoplasm & Xanthogranuloma \\
\hline Benign fibrous histiocytoma & Benign fibrous histiocytoma \\
\hline Neurothekeoma & Fibrous histiocytoma \\
\hline Nodular fasciitis & Juvenile xanthogranuloma \\
\hline Infantile myofibromatosis & Juvenile xanthogranuloma \\
\hline Vascular lesion, favouring hemangioma & Aneurysmal fibrous histiocytoma \\
\hline Mesenchymal neoplasm, likely vascular & \\
\hline Vascular lesion & Benign fibrous histiocytoma/capillary hemangioma \\
\hline Benign to intermediate, 1 case & Dermatofibrosarcoma protuberans \\
\hline Dermatofibroma & \\
\hline Intermediate to benign, 3 cases & Deep dermatofibroma \\
\hline Dermatofibrosarcoma protuberans & Cellular dermatofibroma \\
\hline $\begin{array}{l}\text { Dermatofibrosarcoma protuberans } \\
\text { Smooth muscle tumor of uncertain malignant potential }\end{array}$ & Cellular fibrous histiocytoma \\
\hline
\end{tabular}

TABLE 8: Changes in assignment of tumor behavior.

\begin{tabular}{lcccc}
\hline Category & Number of cases & Major discrepancy & Minor discrepancy & No impact on management/not comparable \\
\hline Malignant to malignant & 99 & 75 & 18 & 6 \\
Malignant to benign & 12 & 11 & 1 & \\
Benign to malignant & 20 & 20 & 12 & 26 \\
Malignant to intermediate & 11 & 11 & 5 \\
Benign to benign & 39 & 1 & 4 & 1 \\
Benign to intermediate & 6 & 12 & & \\
Intermediate to benign & 18 & 11 & 40 & 33 \\
Intermediate to malignant & 15 & 1 & \\
Intermediate to intermediate & 1 & 148 & & \\
Total & 221 & &
\end{tabular}

Table 9: Percentage of diagnostic concordance according to type of referring institution.

\begin{tabular}{lcc}
\hline Type of hospital & Number of cases & \% of concordance \\
\hline Academic/teaching centers & $285(49 \%)$ & 173 cases $(60 \%)$ \\
Community (district general) hospitals & $242(42 \%)$ & 131 cases $(54 \%)$ \\
Private institutions & $38(6 \%)$ & 23 cases $(60 \%)$ \\
Overseas & $16(3 \%)$ & 11 cases $(69 \%)$ \\
\hline
\end{tabular}

(Table 1). Of the nine cases of melanoma/clear cell sarcoma with diagnostic discrepancy, one case had an original diagnosis of Spitz nevus, one was of leiomyosarcoma, and in seven cases a wide range of differentials was offered as the original diagnosis. Amongst seven cases of mesothelioma with diagnostic discrepancy, four had an original diagnosis of carcinoma, two of poorly differentiated small cell sarcoma, and one case from the pleura had a diagnosis of synovial sarcoma. In 15 cases where a second opinion of poorly differentiated carcinoma was given, 13 were initially diagnosed as mesenchymal neoplasms, one as a poorly differentiated epithelioid neoplasm, and one as epithelioid hemangioendothelioma. For certain lesions (e.g., pericytic tumors, myoepithelial tumors, angiomatoid fibrous histiocytoma, acral fibromyxoma, and genital stromal mesenchymal tumors), the high level of diagnostic discrepancy could be due to the lack of familiarity of general pathologists for these neoplasms, as well as their overlapping morphology with other tumors. The 24 miscellaneous benign nonneoplastic cases $(4 \%)$ on second opinion included organizing hematoma (2), scar tissue (1), fibrosis (5), fat necrosis (1), granulation tissue (5), cutaneous ossification (1), benign cyst (2), and five cases for which it was uncertain as to whether a specific lesion was represented. Only two of this group 
TABLE 10: Tumor groups causing major differences in opinion/difficulties between referral and consultation opinion.

\begin{tabular}{lccc}
\hline & 2009 & 2014 & 2012 (current) \\
\hline Type of cases & Referral & Referral & Second opinion (consultation) \\
Number of specimens & 349 & 350 & 581 \\
\% of discrepancy & $26.6 \%$ & $28.2 \%$ & $38 \%$ \\
Major & $11 \%$ & $16.4 \%$ & $25 \%$ \\
Minor & $15.6 \%$ & $11.8 \%$ & $7 \%$ (Table 8) \\
Major problem areas & Diagnosis of GIST and & Diagnosis of smooth muscle & Diagnosis of undifferentiated neoplasms, \\
identified & smooth muscle tumors & tumors and GIST & fibrohistiocytic and adipocytic tumors \\
\hline
\end{tabular}

showed diagnostic discrepancies. A case of Wegener's granulomatosis was diagnosed as Kimura disease, and another case of possible adenomatoid tumor was diagnosed as reactive mesothelial proliferation on second opinion.

Referral cases are those referred to the tertiary center for further clinical management. These require mandatory review of the original histology and hence, are typically not as diagnostically challenging as second opinions. In 2009, we assessed diagnostic discrepancies for referred cases from 349 specimens over one year and found a total discrepancy rate of $26.6 \%$ including 93 cases of which 38 (11\%) were major and $55(15.6 \%)$ were minor discrepancies [4]. The largest group of discrepancy in 2009 was malignant to malignant diagnoses (15 cases) and the most discrepant diagnosis was for gastrointestinal stromal tumor (GIST) (seven cases), with the main reason being the misinterpretation of CD117 immunohistochemistry. The next groups of major discrepancy were smooth muscle and adipocytic tumors and then grading discrepancies. 203 (58\%) were from district general (community) hospitals, 120 (34\%) were from teaching hospitals, and $26(8 \%)$ were from overseas university hospitals. In 2014, a similar study assessed 350 referral specimens over one year [5]. There was diagnostic agreement of $71.8 \%$, with minor discrepancy rate of $11.8 \%$, including grading discrepancies and $16.4 \%$ major discrepancies. There were seven cases of GIST and 19 smooth muscle tumors with discrepancies, although only five adipocytic tumors had diagnostic discrepancy. 230 (66\%) were from district general hospitals, 83 (24\%) were from teaching hospitals, 8 (2\%) were from private laboratories, and $27(8 \%)$ were from overseas.

The numbers of second opinion cases for the one-year period seen in this study are significantly greater than the numbers seen in the previous referral case audits (Table 10). This would reflect the increasing overall workload at this tertiary center and possibly also might be due to increasing subspecialization of pathologists, leading to more requests for second-opinion diagnoses for putative soft tissue tumors. There was overall diagnostic agreement of $73 \%$ and $71.8 \%$ in the previous referral studies compared to $62 \%$ in this audit, in line with the greater diagnostic complexity of secondopinion cases. This comprised a large group of 89 cases where it was difficult even to determine the tumor lineage, and these were often described as undifferentiated malignant neoplasms or classified by their predominant morphologic features, for example, undifferentiated pleomorphic, spindle cell, or small-cell sarcomas. The majority of cases $(49 \%)$ in this study were from teaching hospitals and $42 \%$ from community hospitals (Table 9). This might be due to more complex cases being treated by larger teaching hospitals in the UK. In all these studies, there was no correlation between the incidence of discrepancy and the type of referring institution. In this study, 148 cases $(25 \%)$ were termed major discrepancies and included discrepancies where tumors were placed in different categories of benign/intermediate/ malignant behavior, considerably changing management (Tables 2-8). This higher percentage of major discrepancies in this audit, compared to 11 and $16.4 \%$ in the previous audits for referral cases, emphasizes the importance of obtaining expert second opinion for soft tissue neoplasms. 40 cases $(7 \%)$ were considered as minor discrepancies leading to minor management change, while for 33 cases (5\%), although there was a change in the diagnosis, this did not impact further management.

There were nine diagnoses of GIST in this study after second opinion (1.5\%), and diagnostic discrepancy was noted in four; in these, the diagnosis was changed from mesothelioma (on morphology), SFT (DOG1 positive but no KIT or PDGFRA mutations on second-opinion workup), "vascular lesion" (DOG1 negative, PDGFRA mutation positive), and leiomyoma (KIT mutation positive). This indicates that although GIST is one of the commonly seen tumors in referral practice, it is not often sent for second opinion; these do not seem to pose a diagnostic challenge because of the awareness for GIST amongst general pathologists. Similarly, in this study, smooth muscle tumors also showed a lower discrepancy rate when compared to the fibrohistiocytic and adipocytic tumors. Although the fibroblastic/myofibroblastic tumors formed the largest group of tumors sent for second opinion, they overall showed a lower discrepancy rate when compared to other groups of tumors.

In terms of the utility of immunohistochemistry in second-opinion diagnoses, spindle cell neoplasms were found to be particularly challenging due to the overlap in their morphologic features, and the fact that some sarcomas such as low-grade fibromyxoid sarcoma (LGFMS) have typically bland cell morphology and can be missed if diagnostic awareness is lacking. MUC4 was performed in 20 myxoid spindle cell neoplasms, of which for six this helped towards the diagnosis of LGFMS. Of 13 cases of fibromatosis, seven were confirmed with positive $\beta$-catenin immunohistochemistry. Conversely, fibromatosis can be overcalled, as $\beta$-catenin can be focally positive in scar tissue, which was seen in one case. Amongst nine cases of synovial sarcoma, TLE1 was positive in seven. TLE1 was used in 14 
other cases and found to be focally positive in other tumors including MPNST, carcinoma, and SFT. In two cases of undifferentiated carcinoma, INI1 was positive and useful in excluding epithelioid sarcoma, whilst in two cases the absence of nuclear INI1 was used to confirm the diagnosis of epithelioid sarcoma. Nevertheless, most diagnoses were overturned based on morphologic grounds alone.

In terms of the utility of molecular genetic testing, this was performed in 190 cases (32\%), with test selection dependent on tumor morphology and immunophenotype. For the large group of undifferentiated malignant neoplasms described earlier (Table 1), a wider panel of molecular testing was used to exclude specific entities before putting them under the category of undifferentiated neoplasms. For example, in 16 such cases, RT-PCR for EWSR1 fusion transcripts was performed in 10, fluorescence in situ hybridization (FISH) for MDM2 amplification in four, molecular investigations for SS18-SSX1/2 translocations in four, $P A X-3 / 7-F O X O 1$ translocations in two, BRAF and KIT mutational analysis in two, and PDGFRA mutational analysis in one.

Amongst tumors with predominant spindle cell morphology, molecular testing was used in 62 cases. The various tests were for exclusion of myxoid liposarcoma with FUSDDIT3 fusion transcripts with RT-PCR (one case), MDM2 gene amplification with FISH (negative in all three cases), FUS-CREB3L1/2 fusion transcripts with RT-PCR (13 cases, for which diagnosis of LGFMS was confirmed in four), various EWSR1 fusion transcripts with RT-PCR (negative in all 11 cases), $A L K$ gene rearrangements (11 cases, with confirmation of a diagnosis of inflammatory myofibroblastic tumor (IMT) in three), KIT mutational analysis (13 cases of which GIST was confirmed in four), PDGFRA mutational analysis (10 cases of which GIST was confirmed in one), JAZF1-SUZ12 fusion transcripts (negative in all three cases), and SS18-SSX1/2 fusion transcripts (17 cases). Of the latter, seven cases of synovial sarcoma with spindle cell morphology were confirmed with positive molecular results. Another well-documented diagnostically challenging entity is nodular fasciitis which often leads to confusion with sarcoma and other myofibroblastic proliferations, especially as immunohistochemistry (typically SMA expression alone) is not always helpful. We had two such cases for which the original diagnosis was low-grade spindle cell tumor and atypical nodular spindle cell lesion, with review diagnosis changed to nodular fasciitis based on morphologic appearances. Previous studies have shown that for difficult cases, FISH analysis for USP6 gene rearrangement is useful, with detection in $74.4 \%$ [15]. One case of ossifying fasciitis was positive for USP6 gene fusion with FISH in our study. A case of spindle cell tumor NOS was confirmed as angiomatoid fibrous histiocytoma due to the finding of EWSR1CREB1 fusion transcripts with RT-PCR.

In tumors with small round cell morphology, amongst nine cases of rhabdomyosarcoma, in eight, RT-PCR for $P A X 3 / 7-F O X O 1$ fusion transcripts was performed and was positive in one case, diagnosed as alveolar rhabdomyosarcoma. Three cases of Ewing sarcoma were confirmed by the finding of EWSR1-FLI1 fusion transcripts with RT-PCR. In two cases of poorly differentiated synovial sarcoma with round cell morphology, the diagnosis was made based on positive SS18-SSX1/2 fusion transcripts, and two cases were also positive for TLE1.

Adipocytic neoplasms represent one of the most diagnostic challenging soft tissue tumors for general pathologists. FISH for $M D M 2$ amplification status was performed in $61 / 74$ cases of adipocytic neoplasms and was found to be the most useful technique for confirming or excluding a diagnosis of well-differentiated liposarcoma (WDL) or dedifferentiated liposarcoma (DDL). This test failed in three cases. Nine cases of lipoma were received with a request to exclude WDL, of which FISH for MDM2 amplification was performed in eight and found to be negative. One spindle cell lipoma was diagnosed on morphologic grounds alone. In 26 cases, MDM2 FISH helped to confirm the diagnosis of liposarcoma, with 16 WDL and 10 cases of DDL, all of which were intra-abdominal. Of the four tumors originally thought to be lipomas which turned out to be WDLs with FISH, for two cases the original diagnosis was of pleomorphic lipoma, highlighting the difficulty in interpretation of pleomorphic floret type-cells. Of the seven cases initially diagnosed as WDL which turned out to be benign, three were lipomas with fat necrosis, with inflammatory cells and fibrosis surrounding fat necrosis being misinterpreted as atypical stromal cells and the fibrous septa of WDL. As differentiated adipocytic lesions can pose a diagnostic challenge even to specialist soft tissue pathologists and MDM2 amplification with FISH represents the diagnostic gold standard in distinguishing WDL from benign adipocytic lesions [16-18], there should be a low threshold for performing FISH for MDM2 amplification. One case was originally reported as nondiagnostic then diagnosed as DDL based on the finding of MDM2 amplification with FISH on second opinion. RTPCR for FUS-DDIT3 gene fusion was performed in one case of unclassifiable adipocytic tumor and in two cases of lipoblastoma. In 18 cases, CDK4 immunohistochemistry was also performed and correlated with MDM2 FISH results in 16 but was contradictory in two. p16 was performed in five cases along with CDK4, but CDK4 was more useful in adipocytic tumors as p16 can also be positive in areas of fat necrosis.

Molecular diagnosis was utilized in 14 cases initially diagnosed as melanoma. Two cases were received with request for $B R A F$ mutational analysis, which was detected in one case. In seven, analysis for EWSR1-CREB1 and EWSR1$A T F 1$ fusion transcripts or EWSR1 gene rearrangement was performed to exclude clear cell sarcoma (CCS). Two diagnoses were changed to CCS based on positive molecular findings, while in the remaining five cases mutational analysis for $B R A F$ was performed, with confirmation as melanoma in three where the mutation was detected. In one case, the material was insufficient, and the other case was still diagnosed as melanoma morphologically. In one case of malignant PEComa, BRAF and KIT mutations were found to be negative, with melanoma excluded. In 6 cases of carcinomas with sarcomatoid or epithelioid morphology, SS18SSX1/2 was useful in excluding synovial sarcoma. In three cases of mesothelioma, in two where the differential 
diagnosis was of synovial sarcoma, SS18-SSX1/2 molecular analysis was performed, and in one case FISH for $A L K$ gene rearrangement was performed to exclude IMT. In one case of chondroma, RT-PCR for EWSR1-NR4A3 gene fusion was performed to exclude extraskeletal myxoid chondrosarcoma. In three cases of myoepithelioma, FISH for EWSR1 gene rearrangement was performed, but was negative in all, and the diagnosis was made on morphology alone.

As it is evident from the discussion, diagnosis in most consultation cases was largely done based on morphology alone by specialist soft tissue pathologists, with subsequent selection of an appropriate confirmatory immunopanel, and ancillary molecular investigations were often as useful in the exclusion of specific diagnoses as they were in confirmation. Confirmatory molecular investigations for specific neoplasms are important for the use of targeted therapies such as BRAF or CDK4 inhibitors (in confirming that patients have melanoma rather than CCS and for patients with DDL, respectively) and for patient enrolment into appropriate clinical trials. Furthermore, there is increasing recognition of the importance of histology-tailored therapy in treating soft tissue sarcomas, making accurate histologic diagnosis fundamental to treatment selection [19]. This has been highlighted by the introduction of tyrosine kinase inhibitors in GIST, including imatinib, sunitinib, and regorafenib. However, certain GIST mutations are known to be resistant to these agents (PDGFRA D842V), and there are new agents available in clinical trials that have shown efficacy against these treatment-resistant subtypes [20]. This highlights the importance of expert multidisciplinary care for patients with rare cancers. In addition, there are a number of ongoing subtype-specific trials in various sarcoma subtypes including epithelioid sarcoma, angiosarcoma, undifferentiated pleomorphic sarcoma, and liposarcoma. Accurate histopathological diagnosis is also of critical importance not only for discussing treatment options but also for prognosis with patients and their families.

In summary, the diagnostic discrepancy rate of $38 \%$ for second-opinion cases highlights the importance of obtaining opinions from specialist soft tissue pathologists in challenging cases, especially when working in centers where newer antibodies and molecular testing are not available. Obtaining an accurate diagnosis by second opinion is essential due to the diagnostic implications on management.

\section{Data Availability}

Data are available upon request from the corresponding author.

\section{Conflicts of Interest}

The authors declare that they have no conflicts of interest regarding the publication of this paper.

\section{Authors' Contributions}

All authors contributed equally to this manuscript.

\section{References}

[1] https://www.nice.org.uk/guidance/csg9/resources/improvingoutcomes-for-people-with-sarcoma-update-773381485.

[2] C. Fisher, Standards and Datasets for Reporting Cancers. Dataset for the Histopathological Reporting of Soft Tissue Sarcomas, Royal College of Pathologists, London, UK, 2017.

[3] C. D. M. Fletcher, J. A. Bridge, P. C. W. Hogendoorn, and F. Mertens, World Health Organization Classification of Tumors of Soft Tissue and Bone, IARC, Lyon, France, 4th edition, 2013.

[4] K. Thway and C. Fisher, "Histopathological diagnostic discrepancies in soft tissue tumors referred to a specialist centre," Sarcoma, vol. 2009, Article ID 741975, 2009.

[5] K. Thway, J. Wang, T. Mubako, and C. Fisher, "Histopathological diagnostic discrepancies in soft tissue tumors referred to a specialist centre: reassessment in the era of ancillary molecular diagnosis," Sarcoma, vol. 2014, Article ID 686902, 2014.

[6] Z. K. Arbiser, A. L. Folpe, and S. W. Weiss, "Consultative (expert) second opinions in soft tissue pathology," American Journal of Clinical Pathology, vol. 116, no. 4, pp. 473-476, 2001.

[7] M. Lehnhardt, A. Daigeler, J. Hauser et al., "The value of expert second opinion in diagnosis of soft tissue sarcomas," Journal of Surgical Oncology, vol. 97, no. 1, pp. 40-43, 2008.

[8] A. Al-Ibraheemi and A. L. Folpe, "Voluntary second opinions in pediatric bone and soft tissue pathology," International Journal of Surgical Pathology, vol. 24, no. 8, pp. 685-691, 2016.

[9] L. Perrier, P. Rascle, M. Morelle et al., "The cost-saving effect of centralized histological reviews with soft tissue and visceral sarcoms, GIST, and desmoid tumors: the experiences of the pathologists of the French Sarcoma Group," PLOS one, vol. 13, no. 4, pp. 1-22, 2018.

[10] W. J. Anderson and J. L. Hornick, "Immunohistochemical correlates of recurrent genetic alterations in sarcomas," Genes, Chromosomes and Cancer, vol. 58, no. 2, pp. 111-123, 2019.

[11] J. L. Hornick, "Novel uses of immunohistochemistry in the diagnosis and classification of soft tissue tumors," Modern Pathology, vol. 27, no. S1, pp. S47-S63, 2014.

[12] J. A. Bridge, "Advantages and limitations of cytogenetic, molecular cytogenetic, and molecular diagnostic testing in mesenchymal neoplasms," Journal of Orthopaedic Science, vol. 13, no. 3, pp. 273-282, 2008.

[13] J. M. Coindre, M. Trojani, G. Contesso et al., "Reproducibility of a histopathologic grading system for adult soft tissue sarcoma," Cancer, vol. 58, no. 2, pp. 306-309, 1986.

[14] L. Guillou, J. M. Coindre, F. Bonichon et al., "Comparative study of the national cancer institute and french federation of cancer centers sarcoma group grading systems in a population of 410 adult patients with soft tissue sarcoma," Journal of Clinical Oncology, vol. 15, no. 1, pp. 350-362, 1997.

[15] R. Erber and A. Agaimy, "Misses and near misses in diagnosing nodular fasciitis and morphologically related reactive myofibroblastic proliferations: experience of a referral center with emphasis on frequency of USP6 gene rearrangements," Virchows Archiv, vol. 473, no. 3, pp. 351-360, 2018.

[16] K. Thway, J. Wang, J. Swansbury, T. Min, and C. Fisher, "Fluorescence in situ hybridization for MDM2 amplification as a routine ancillary diagnostic tool for suspected well-differentiated and dedifferentiated liposarcomas: esperience at a tertiary center," Sarcoma, vol. 2015, Article ID 812089, 2015.

[17] E. G. Demicco, "Molecular updates in adipocytic neoplasms," Seminars in Diagnostic Pathology, vol. 36, no. 2, pp. 85-94, 2019. 
[18] J. Weaver, P. Rao, J. R. Goldblum et al., "Can MDM2 analytical tests performed on core needle biopsy be relied upon to diagnose well-differentiated liposarcoma?" Modern Pathology, vol. 23, no. 10, pp. 1301-1306, 2010.

[19] J. Noujaim, K. Thway, A. Sheri, C. Keller, and R. L. Jones, "Histology-driven therapy," International Journal of Surgical Pathology, vol. 24, no. 1, pp. 5-15, 2016.

[20] Z. Szucs, K. Thway, C. Fisher et al., "Promising novel therapeutic approaches in the management of gastrointestinal stromal tumors," Future Oncology, vol. 13, no. 2, pp. 185-194, 2017. 